\title{
Development and Validation of the B Cell-Associated Fc Receptor- like Molecule-Based Prognostic Signature in Skin Cutaneous Melanoma
}

\author{
Yu Liu, ${ }^{1}$ Yiding Chen $\left(\mathbb{D},{ }^{2}\right.$ Xianyu Hu, ${ }^{3}$ Jialin Meng $\mathbb{D}^{2},{ }^{2}$ and Xiaojing Li $\mathbb{D}^{1}$ \\ ${ }^{1}$ Department of Plastic Surgery, First Affiliated Hospital of Anhui Medical University, Hefei, Anhui, China \\ ${ }^{2}$ Department of Urology, The First Affiliated Hospital of Anhui Medical University; Institute of Urology \& Anhui Province Key \\ Laboratory of Genitourinary Diseases, Anhui Medical University, Hefei, Anhui, China \\ ${ }^{3}$ Department of General Surgery, The First Affiliated Hospital of Anhui Medical University, Hefei, Anhui, China
}

Correspondence should be addressed to Jialin Meng; mengjialin@ahmu.edu.cn and Xiaojing Li; ay_lxj@yahoo.com

Received 21 March 2020; Revised 8 July 2020; Accepted 3 August 2020; Published 24 August 2020

Academic Editor: Adam Reich

Copyright (C) $2020 \mathrm{Yu}$ Liu et al. This is an open access article distributed under the Creative Commons Attribution License, which permits unrestricted use, distribution, and reproduction in any medium, provided the original work is properly cited.

Backgroud. Cutaneous melanoma (CM) causes the highest absolute number of deaths among all types of skin cancers; however, the association between Fc receptor- (FcR-) like (FCRL) molecules and CM remains unclear. Methods. 461 patients with CM from The Cancer Genome Atlast- (TCGA-) CM cohort and 290 pateints from the GSE65904 cohort were enrolled. Student's $t$-test was used to compare the differences, and Pearson's correlation coefficient was employed to evaluate associations. The Kaplan-Meier (K-M) survival analysis was used to evaluate overall survival (OS). The multivariate Cox regression was conducted to generate the FCRL prognostic signature. GSEA analysis and TIMER were employed to study the potential mechanisms. Result. Patients with Breslow's depth high or equal to $3 \mathrm{~cm}$ had the lower expression of FCRL1-6 (all, $P<0.05$ ), which indicates poor OS, as well as age, stage, and Breslow's depth subgroups (all, $P<0.001$ ). The overall FCRL1-6 prognostic signature was generated in the TCGA cohort (K-M, $P<0.001$; area under the curve (AUC), 0.649 for 3-year OS) and validated in the GSE65904 cohort (K-M, $P<0.001$; AUC, 0.659 for 3-year OS). The GSEA results revealed that high expression of FCRLs indicated activated immune-associated pathways, and FCRLs are positively associated with the infiltration of B cells. Conclusion. Highly expressed FCRLs were observed associated with a favourable OS of CM. FCRL1-6-based prediction signature could act as a biomarker to predict the prognosis of patients with CM.

\section{Introduction}

Cutaneous melanoma $(\mathrm{CM})$ causes the highest absolute number of deaths among all skin tumors and consists of four main types: lentigo malignant melanoma, nodular melanoma, acral lentiginous melanoma, and superficial spreading malignant melanoma $[1,2]$. Globally, melanoma accounted for $1.6 \%$ of new cancer cases and caused $0.6 \%$ of cancerspecific deaths in 2018 [3]. Furthermore, the mortality of CM has increased sharply in the past decades: The median mortality of CM was $\sim 1.55$ per 10,000 individuals for males in 1985-1987 and increased to 2.57 in 2013-2015, while the median mortality of females also increased from 1.39 to 1.55 [4]. In the USA, there were $\sim 1,000,000$ new cases and
7,000 CM-related death in 2019, and both of them are higher in males compared with those in females [5]. Several factors have been reported to affect the risk of CM; among them, ultraviolet (UV) light plays a key role [6], especially indoor solar UV exposure, which leads to high UV exposure and depletion of vitamin D3. Sunburn, cosmetic ingredients, sex hormones, family history, and DNA mutations are all risk factors for CM [7].

The association between defects in the immune system activity and CM has been evaluated by several studies, which demonstrated that alterations in immune cells and proteins are potential factors for immunosurveillance and progression of CM [8-10]. Park et al. showed that tissue-resident memory $\mathrm{CD} 8+\mathrm{T}$ cells could promote a durable melanoma- 

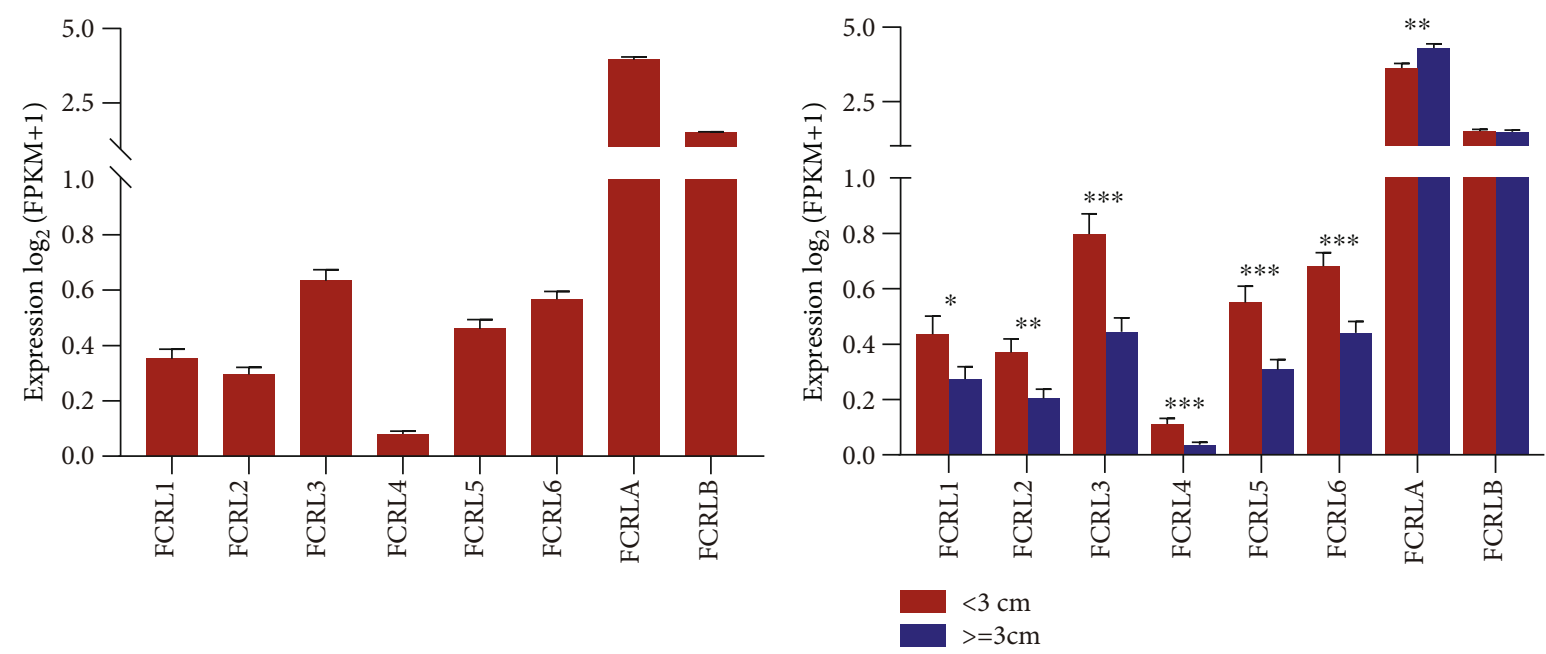

(a)

(b)

\begin{tabular}{|c|c|c|c|c|c|c|c|c|c|}
\hline & mut & cg22515937 & $\operatorname{cg} 24344394$ & $\operatorname{cg} 05766896$ & cg09340639 & $\operatorname{cg} 08933467$ & $\operatorname{cg} 00394221$ & $\operatorname{cg} 11810135$ & \\
\hline \multirow[t]{2}{*}{ FCRL1 } & -0.056 & 0.194 & 0.242 & -0.073 & 0.111 & 0.141 & 0.413 & 0.387 & \\
\hline & mut & $\operatorname{cg} 04559323$ & $\operatorname{cg} 15761405$ & $\operatorname{cg} 05784088$ & cg20674248 & $\operatorname{cg} 12282588$ & cg05777583 & & \\
\hline \multirow[t]{2}{*}{ FCRL2 } & -0.016 & 0.097 & 0.242 & 0.185 & 0.191 & 0.146 & 0.2 & & \\
\hline & mut & $\operatorname{cg} 24881910$ & $\operatorname{cg} 15202552$ & $\operatorname{cg} 05384663$ & $\operatorname{cg} 15602298$ & $\operatorname{cg} 08786003$ & $\operatorname{cg} 17134153$ & $\operatorname{cg} 25259754$ & $\operatorname{cg} 07184316$ \\
\hline \multirow[t]{4}{*}{ FCRL3 } & 0.002 & 0.033 & -0.010 & 0.035 & 0.096 & 0.026 & 0.010 & -0.009 & 0.034 \\
\hline & & $\operatorname{cg} 05956521$ & $\operatorname{cg} 11150520$ & & & & & & \\
\hline & & 0.055 & 0.017 & & & & & & \\
\hline & mut & $\operatorname{cg} 16408815$ & $\operatorname{cg} 17281600$ & $\operatorname{cg} 01612158$ & $\operatorname{cg} 13380622$ & $\operatorname{cg} 18268488$ & & & \\
\hline \multirow[t]{2}{*}{ FCRL4 } & 0.034 & 0.043 & 0.057 & 0.013 & 0.061 & 0.055 & & & \\
\hline & mut & $\operatorname{cg} 11080915$ & $\operatorname{cg} 21443284$ & cg00248302 & cg27578760 & $\operatorname{cg} 14833337$ & $\operatorname{cg} 10313167$ & $\operatorname{cg} 00858294$ & cg20703895 \\
\hline \multirow[t]{2}{*}{ FCRL5 } & 0.011 & 0.453 & 0.442 & 0.186 & 0.143 & 0.307 & -0.009 & 0.074 & 0.050 \\
\hline & mut & $\operatorname{cg} 10016364$ & $\operatorname{cg} 14217495$ & cg06997008 & $\operatorname{cg} 16272007$ & cg24833981 & cg25698205 & $\operatorname{cg} 00936728$ & cg17421805 \\
\hline \multirow[t]{2}{*}{ FCRL6 } & -0.012 & -0.589 & -0.42 & 0.028 & 0.069 & 0.214 & -0.097 & -0.052 & 0.238 \\
\hline & mut & cg24664855 & $\operatorname{cg} 05033369$ & $\operatorname{cg} 24074594$ & $\operatorname{cg} 00714874$ & $\operatorname{cg} 18884942$ & & & \\
\hline \multirow[t]{2}{*}{ FCRLA } & -0.094 & -0.417 & -0.15 & -0.266 & -0.311 & -0.056 & & & \\
\hline & mut & $\operatorname{cg} 22026255$ & $\operatorname{cg} 22678092$ & $\operatorname{cg} 17036419$ & $\operatorname{cg} 00160981$ & $\operatorname{cg} 26015133$ & cg27495845 & cg18889147 & $\operatorname{cg} 14210287$ \\
\hline \multirow[t]{5}{*}{ FCRLB } & -0.069 & -0.159 & -0.219 & -0.298 & -0.265 & -0.214 & 0.204 & -0.156 & -0.244 \\
\hline & & $\operatorname{cg} 21864996$ & $\operatorname{cg} 15642792$ & cg22854981 & $\operatorname{cg} 05392764$ & $\operatorname{cg} 01194901$ & cg22389730 & cg17905084 & $\operatorname{cg} 07385423$ \\
\hline & & -0.102 & -0.044 & -0.041 & -0.106 & -0.058 & -0.075 & -0.102 & -0.287 \\
\hline & & $\operatorname{cg} 11141696$ & $\operatorname{cg} 18200075$ & $\operatorname{cg} 18450905$ & cg26009555 & & & & \\
\hline & & -0.138 & -0.139 & -0.169 & -0.172 & & & Red indicate & $P<0.05$ \\
\hline
\end{tabular}

(c)

FIGURE 1: FCRLs expression in CM patients and the association with promoter DNA methylation. (a) The baseline of eight FCRLs in CM patients in the TCGA-CM cohort. (b) The different expression of FCRLs in patients with high ( $\geq 3 \mathrm{~cm})$ or low $(<3 \mathrm{~cm})$ Breslow's depth. (c) The association of FCRLs and DNA methylation at their promoter region.

immune equilibrium that is confined to the epidermal layer of the skin [11]. Eggermont et al. reported that ipilimumab, at a dose of $10 \mathrm{mg}$ per $\mathrm{kg}$ of body weight, resulted in significantly higher rates of recurrence-free survival, overall survival (OS), and distant metastasis-free survival than those of the placebo in high-risk Stage III melanoma [12]. Fc receptor- (FcR-) like (SRGRV) molecules belong to the immuno- globulin superfamily (IgSF) and have a potential activating and inhibitory role [13]. In humans, this family encodes six transmembrane receptors, FCRL1-6, and two cytoplasmic proteins, including FCRLA and FCRLB that lack any type of transmembrane region [14]. The expression profile of FCRLs has been widely investigated in several malignancies such as chronic lymphocytic leukemia (CLL) and acute 

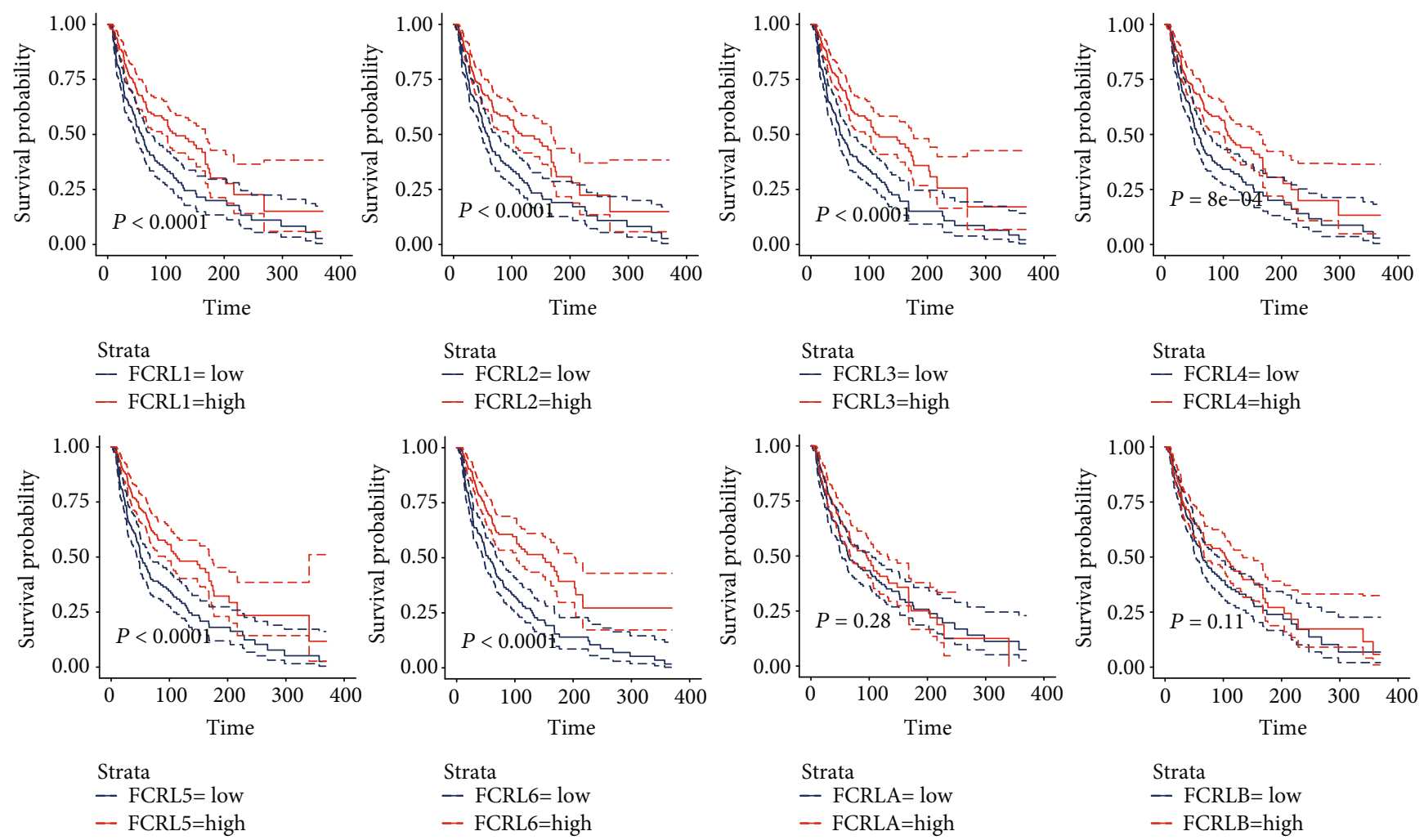

(a)
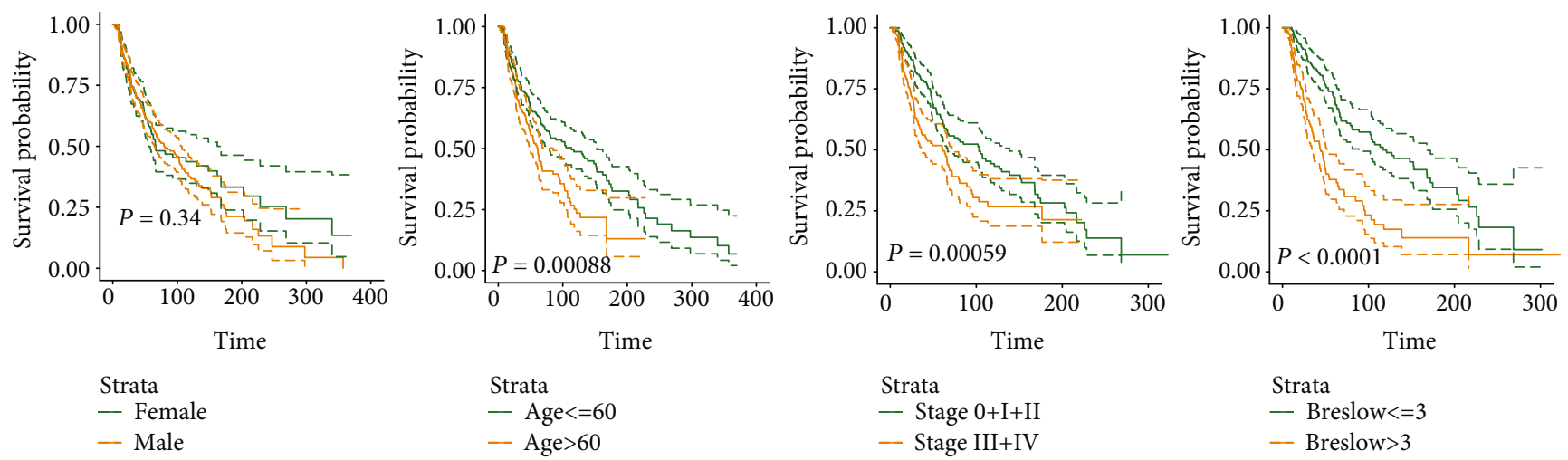

(b)

FIGURE 2: The K-M plot showing the different OS in subgroups. (a) Different OS outcomes in high-and low-expression groups of FCRLs. (b) Different OS outcomes in sex, age, stage, and Breslow's depth subgroups.

lymphocytic leukemia (ALL) [15-17], in infectious diseases such as malaria, hepatitis, and acquired immunodeficiency syndrome [18-20], and in some autoimmune diseases [2123]. It has been reported that FRCL gene expression may be upregulated in the progression of melanoma [24].

However, the mechanism of FCRLs in CM has not been yet elucidated. Therefore, the present study explored the different expression of FCRLs in subgroups of patients with CM and generated and validated the determined prognostic signature. Moreover, Gene Set Enrichment Analysis (GSEA) was employed to identify the key pathways among high-risk and low-risk groups. In addition, immune cell infiltration analysis was performed by the tumor immune microenvironment (TIME) online tool to deeply illustrate the association between FCRLs and the polarized prognosis of patients with CM.

\section{Materials and Methods}

2.1. Study Population. The Cancer Genome Atlas- (TCGA-) CM data were downloaded from UCSC Xena (https://tcga .xenahubs.net), including the Illumina HiSeq pan-cancer normalized gene expression data and the DNA methylation data from the HumanMethylation450 platform. An external validation cohort, GSE65904, was downloaded from the Gene Expression Omnibus database (https://www.ncbi.nlm.nih.gov/geo/). 

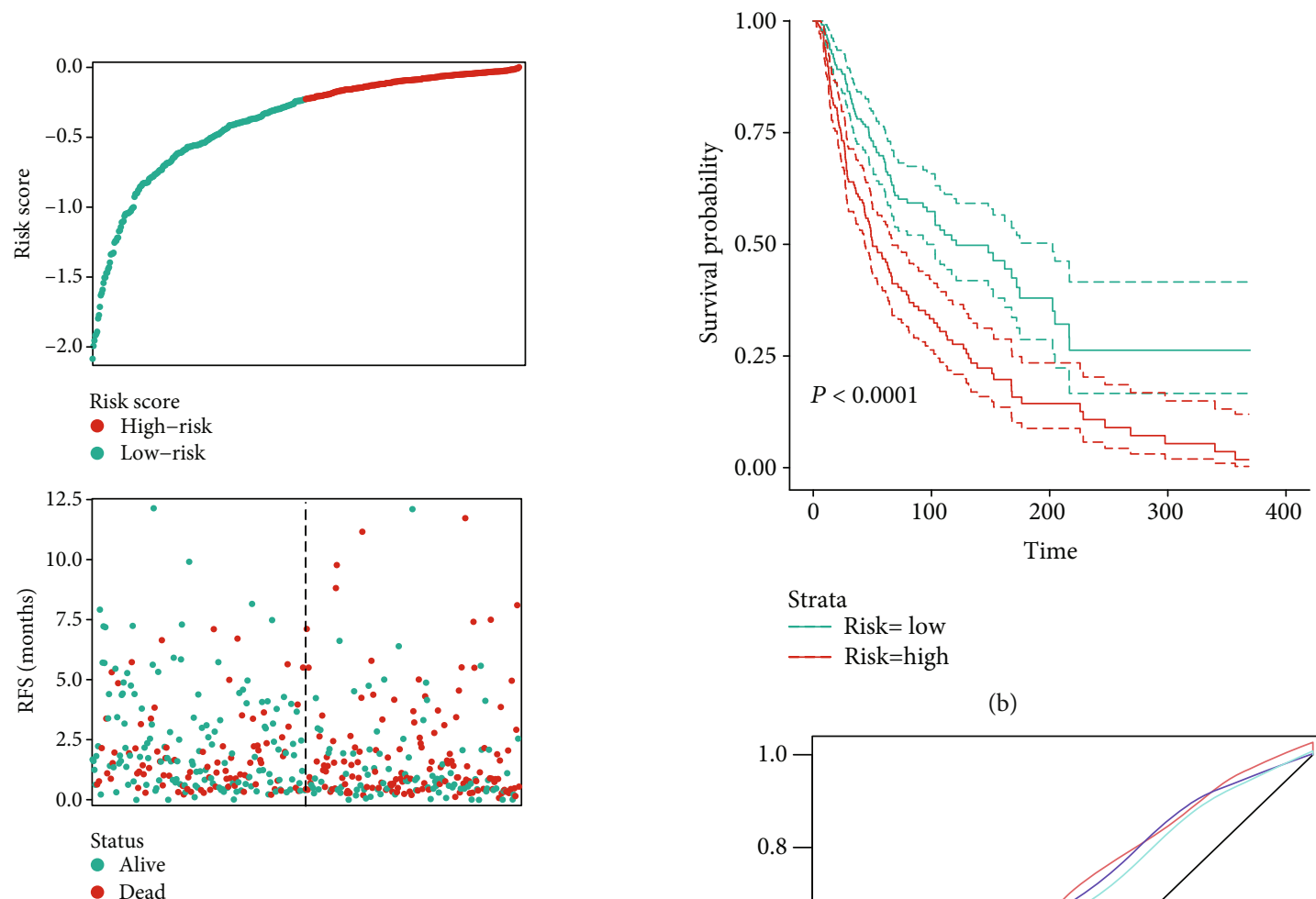

(b)
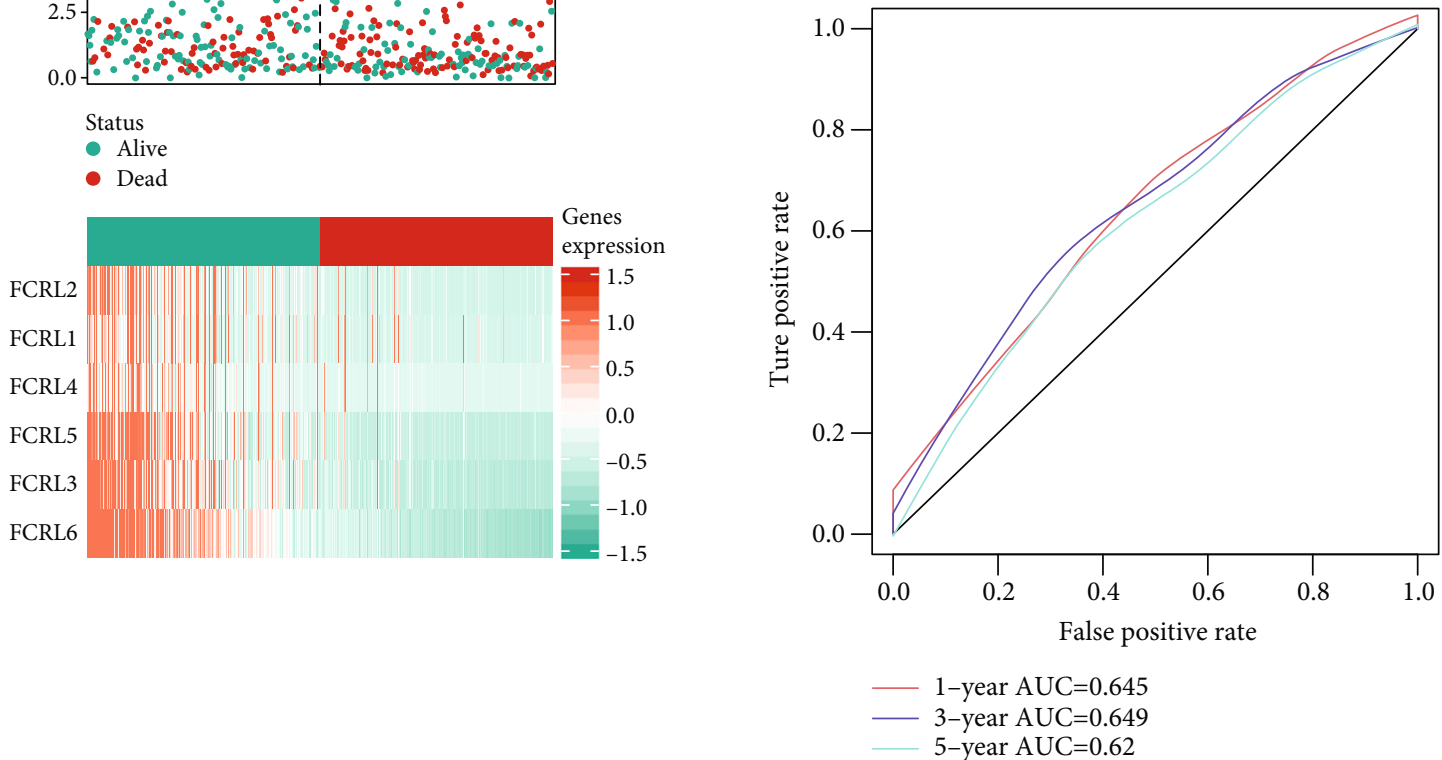

(a)

(c)

FIGURE 3: Establishment of FCRL OS prediction value in the TCGA-CM cohort. (a) Risk score for each patient in TCGA-CM cohort and heatmap to show the expression of FCRL1-6. (b) The K-M plot showing the different OS outcome in high-risk and low-risk groups. (c) ROC curve showing the AUC value in 1-year, 3-year, and 5-year predicted accuracy.

2.2. Statistical Methods. Graphpad 8.0 was used to display the different expression of FCRLs in various subgroups of patients with CM. Student's $t$-test was used to compare the differences between unpaired values, while the Pearson's product-moment correlation coefficient was employed to evaluate the association between $\mathrm{CpG}$ sites and mRNA expression of FCRLs. The Kaplan-Meier (K-M) survival analysis of patient subgroups with high and low levels of FCRLs and clinicopathological characteristics was also performed.

2.3. Establishment and Validation of the Prognostic Signature. To evaluate the overall prognostic value of FCRLs, we generated the FCRLs prognostic signature based on the results of the multivariate Cox regression with the TCGA-CM cohort, and each patient obtained a risk score from the signature. The K-M survival analysis was used to indicate the different outcomes of risk groups with the "survminer" package. Next, the time-dependent receiver operating characteristic (ROC) curve analysis was used to calculate the area under the curve (AUC) for 1-year, 3-year, and 5-year OS and disease-free survival, and to determine the prediction accuracy of our model using the "survivalROC" package. The predicted value and accuracy were also validated in the GSE65904 cohort.

2.4. Gene Set Enrichment Analysis (GSEA) Analysis among FCRLs Determined Risk Groups. GSEA primarily analyzes microarray data, using genomic and genetic sequencing, to detect significant biological differences in microarray 

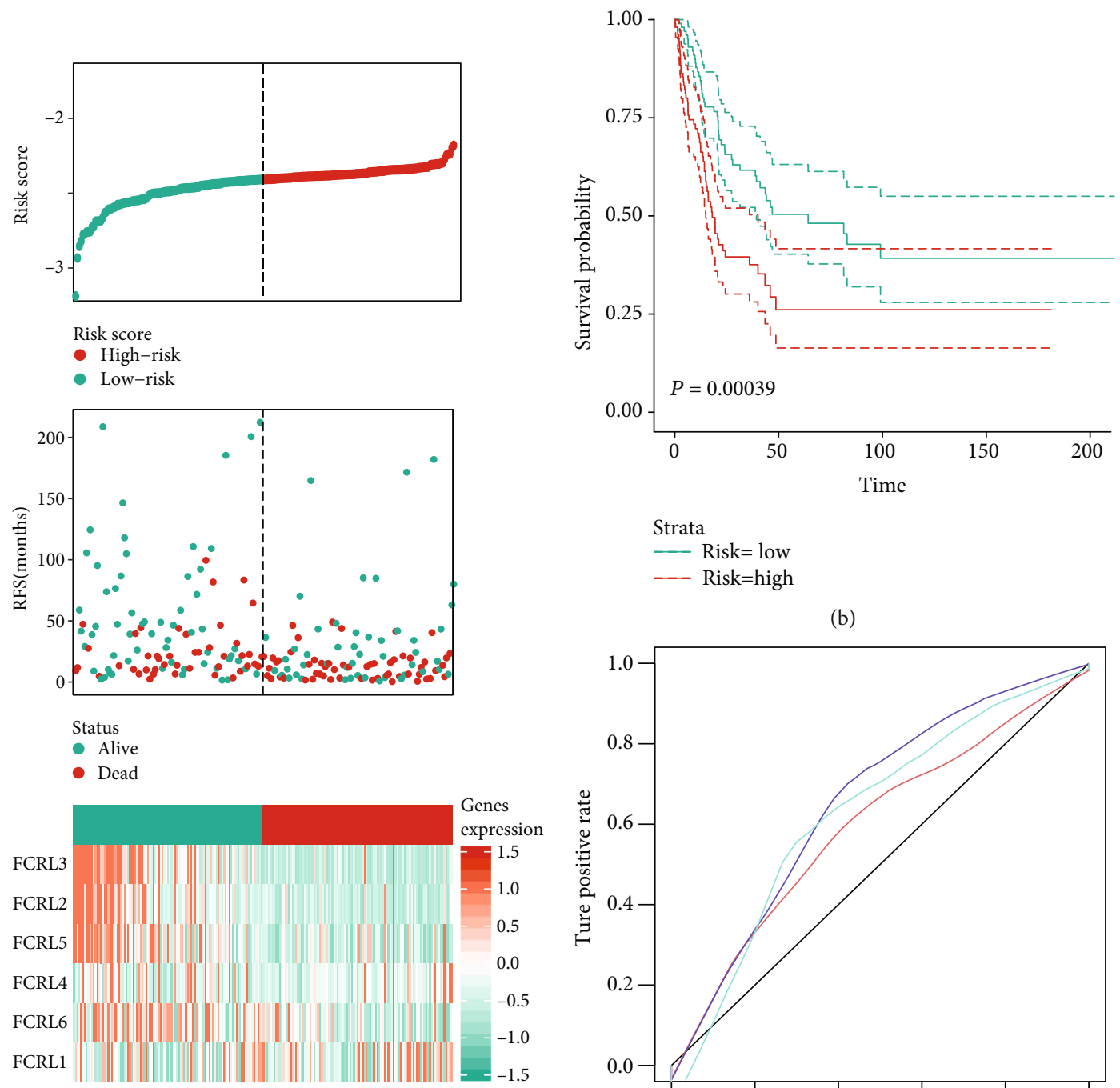

(b)

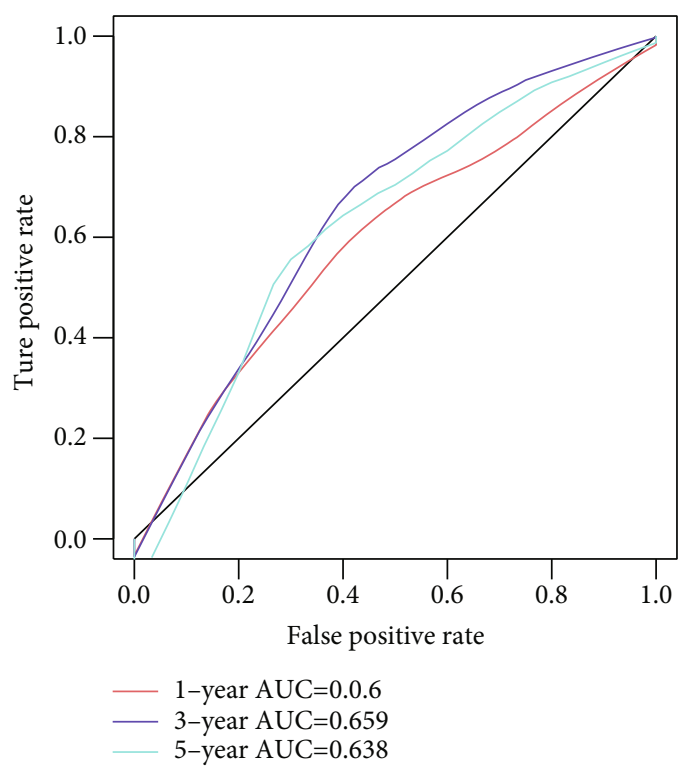

(a)

(c)

FIGURE 4: Validation of FCRL OS prediction value in the GSE65904 cohort. (a) Risk score for each patient in GSE65904 cohort and heatmap to show the expression of FCRL1-6. (b) The K-M plot showing the different OS outcome in high-risk and low-risk groups. (c) ROC curve showing the AUC value in 1-year, 3-year, and 5-year predicted accuracy.

datasets [25]. In the present study, differentially expressed genes and common crucial pathways between FCRLdetermined high- and low-risk groups were identified by GSEA. The datasets were normalized and the intensity of the $\log _{10}$ probe set was calculated using the Robust Multichip averaging algorithm with bioconductors [26]. The pathway analysis of each dataset was performed independently. The variability was measured in the interquartile range (IQR), and a cut-off was set to foreclose IQR values $<0.5$ for all the remaining genes. If one gene was targeted in multiple probe sets, the probe set with the greatest variability was retained. Besides, genes in each pathway were subjected to the statistical analysis system (SAS), and each pathway's
$P$ value was obtained in the permutation test with $\times 1000$. $P<0.05$ was considered to indicate a statistically significant difference.

2.5. TIME Analysis by TIMER. TIME analysis was performed by the TIMER [27] online tool, aimed at deeply illustrating the association between FCRLs and the polarized prognosis of patients with CM. TIMER is a comprehensive resource for systematic analysis of immune infiltrates across diverse cancer types. The abundances of six immunocytes (B cells, CD4+ T cells, CD8+ T cells, neutrophils, macrophages, and dendritic cells) were estimated by the TIMER algorithm. 


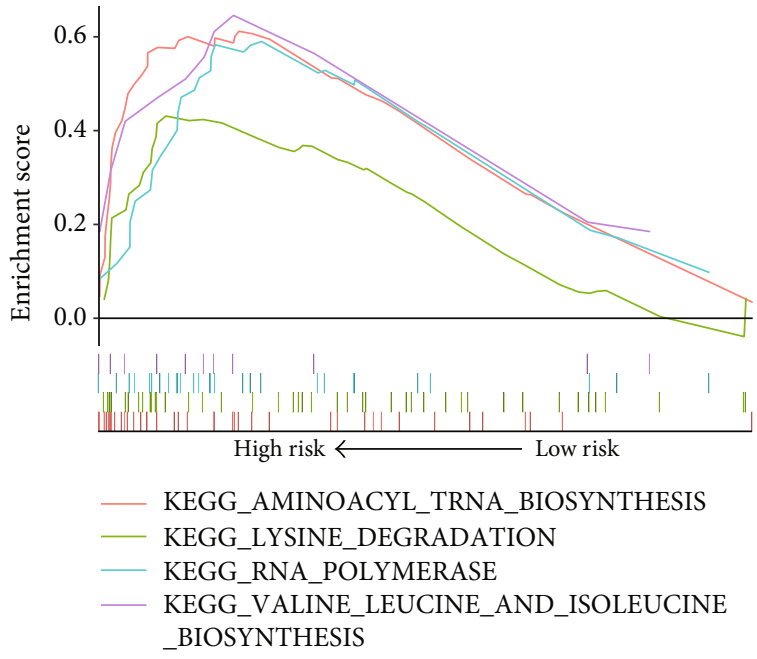

(a)

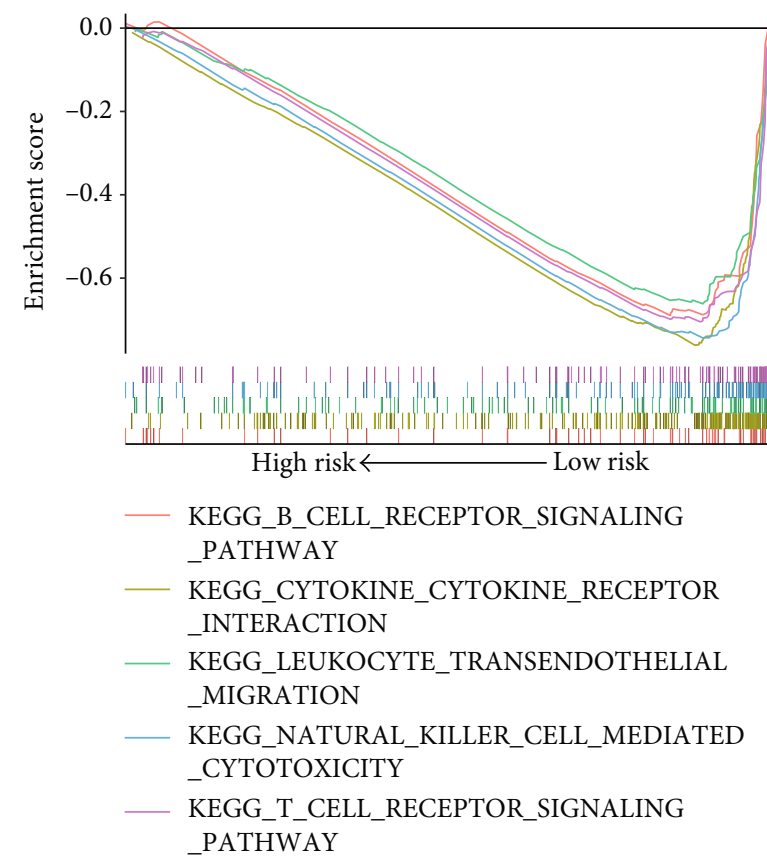

(b)

FIGURE 5: GSEA results showing the highly activated pathways in FCRL-determined high-risk and low-risk groups. (a) The activated signaling pathways in FCRL-determined high-risk group. (b) The activated signaling pathways in FCRL-determined low-risk group.

\section{Results}

3.1. Associations between mRNA Levels, DNA Methylation, and Pathological Features. We firstly checked the baseline mRNA expression of all 8 FCRLs and found that FCRLA and FCRLB had a high level in all FCRLs. As for the analysis of the Breslow's depth, which indicates the depth of tumor cells have invaded the skin, we observed that patients with the Breslow's depth higher or equal to $3 \mathrm{~cm}$ had the lower expression of FCRL1-6 (all, $P<0.05$ ), while FCRLA showed the opposite result $(P<0.05)$ and FCRLB showed no difference (Figure 1(b)). DNA methylation plays an important role as it affects the mRNA level of genes, so we evaluated the associations between DNA methylation and mRNA expression of FCRLs. We found that the cg11080915 had the most prominent effect on the mRNA level of FCRL5 among all the FCRLs, while cg10016364 had the lowest effect on FCRL6 (Figure 1(c)).

3.2. The Prognostic Value of FCRLs and Pathological and Clinical Features. To assess the overall survival (OS) prognostic value of FCRLs, we divided the patients into two groups by the median of each FCRL expression. Interestingly, we found that the lower expression of FCRL1-6 depicted poor OS (all, $P<0.05$, Figure 2(a)), which is consistent with the results shown in Figure 1(b). However, there are no significant results between FCRLA $(P=0.28)$ and FCRLB $(P=0.085)$ to CM OS (Figure 2(a)). What is more, we also evaluated the prognostic value of several pathological and clinical features. We exposed that age $<60$ or $\geq 60$ years old $(P<0.001)$, Stage $0+\mathrm{I}+\mathrm{II}$ or Stage III+IV $(P<0.001)$, and Breslow's depth $<3$ or $\geq 3 \mathrm{~cm}(P<0.001)$ could distinguish the patients to favour- able and poor outcome subgroups; however, the gender could not $(P=0.34)$ (Figure 2(b)).

3.3. The Establishment and Validation of FCRL-Determined Prognostic Signature. The KIRC-CM cohort was used to establish the predicted signature, based on the results of multivariate Cox Regression analysis, the risk score formula risk score $=$ $0.276 * \mathrm{FCRL}_{\exp }-0.356 * \mathrm{FCRL} 2_{\exp }-0.097 * \mathrm{FCRL}_{\exp }+$ $0.5 * \mathrm{FCRL}_{\text {exp }}-0.208 * \mathrm{FCRL}_{\exp }-0.453 * \mathrm{FCRL}_{\exp } \quad$ was used to calculate the risk of each patients. FCRLA and FCRLB were not involved in the signature because they were not associated with the OS (Figure 2(a)). The risk of patients and mRNA expression of FCRLs in the TCGA-CM cohort are shown in Figure 3(a). The K-M plot indicated that the patients with high risk suffered from a poor OS outcome $(P<0.001$, Figure $3(\mathrm{~b}))$, and the AUC value of the ROC curve is meaningful $(1-$ year $\mathrm{AUC}=0.645,3$ - year $\mathrm{AUC}=0.649$, and 5 - year AUC $=0.620$, Figure 3(c)).

The predicted value of FCRL-determined signatures was also validated in the GSE65904 cohort. All the patients were firstly calculated with the risk score (Figure 4(a)). And the $\mathrm{K}-\mathrm{M}$ survival plot revealed that patients with the high risk show a poor prognosis as compared with the low-risk patients $(P<0.001)$ (Figure $4(\mathrm{~b}))$. The AUC value of the ROC curve is meaningful $(1-$ year $\mathrm{AUC}=0.600,3$-year $\mathrm{AUC}=0.659$, and $5-$ year $\mathrm{AUC}=0.638$, Figure $4(\mathrm{c}))$.

3.4. GSEA Analysis to Expose the FCRL-Affected Signaling Pathways. To reveal the mechanism of how FCRLs affected the process of CM, we performed the GSEA analysis to generate the potential signaling pathways. The analysis was completed between the high- and low-risk patients in the 
TABLE 1: The results of GSEA analysis in high- and low-risk groups determined by FCRLs.

\begin{tabular}{lccc}
\hline Pathway & Size & NES & NOM $P$ value \\
\hline High-risk group & & & \\
KEGG_GLYCOSYLPHOSPHATIDYLINOSITOL_GPI_ANCHOR_BIOSYNTHESIS & 25 & 1.997 & $<0.001$ \\
KEGG_RNA_POLYMERASE & 29 & 1.849 & 0.008 \\
KEGG_AMINOACYL_TRNA_BIOSYNTHESIS & 41 & 1.848 & 0.008 \\
KEGG_GLYOXYLATE_AND_DICARBOXYLATE_METABOLISM & 16 & 1.726 & 0.014 \\
KEGG_VALINE_LEUCINE_AND_ISOLEUCINE_BIOSYNTHESIS & 11 & 1.611 & 0.03 \\
KEGG_LYSINE_DEGRADATION & 44 & 1.526 & 0.05 \\
Low-risk group & & & \\
KEGG_CYTOKINE_CYTOKINE_RECEPTOR_INTERACTION & 258 & -2.777 & $<0.001$ \\
KEGG_CHEMOKINE_SIGNALING_PATHWAY & 186 & -2.765 & $<0.001$ \\
KEGG_CELL_ADHESION_MOLECULES_CAMS & 131 & -2.716 & $<0.001$ \\
KEGG_NATURAL_KILLER_CELL_MEDIATED_CYTOTOXICITY & 130 & -2.694 & $<0.001$ \\
KEGG_VIRAL_MYOCARDITIS & 68 & -2.647 & $<0.001$ \\
KEGG_JAK_STAT_SIGNALING_PATHWAY & 151 & -2.634 & $<0.001$ \\
KEGG_ANTIGEN_PROCESSING_AND_PRESENTATION & 79 & -2.578 & $<0.001$ \\
KEGG_LEUKOCYTE_TRANSENDOTHELIAL_MIGRATION & 116 & -2.565 & $<0.001$ \\
KEGG_TOLL_LIKE_RECEPTOR_SIGNALING_PATHWAY & 101 & -2.525 & $<0.001$ \\
KEGG_LEISHMANIA_INFECTION & 69 & -2.483 & $<0.001$ \\
KEGG_T_CELL_RECEPTOR_SIGNALING_PATHWAY & 107 & -2.478 & $<0.001$ \\
KEGG_SYSTEMIC_LUPUS_ERYTHEMATOSUS & 131 & -2.477 & $<0.001$ \\
KEGG_FC_GAMMA_R_MEDIATED_PHAGOCYTOSIS & 95 & -2.468 & $<0.001$ \\
KEGG_HEMATOPOIETIC_CELL_LINEAGE & 85 & -2.458 & $<0.001$ \\
KEGG_NOD_LIKE_RECEPTOR_SIGNALING_PATHWAY & 61 & -2.414 & $<0.001$ \\
KEGG_AUTOIMMUNE_THYROID_DISEASE & 50 & -2.39 & $<0.001$ \\
KEGG_APOPTOSIS & 86 & -2.34 & $<0.001$ \\
KEGG_B_CELL_RECEPTOR_SIGNALING_PATHWAY & 74 & -2.318 & $<0.001$ \\
KEGG_FC_EPSILON_RI_SIGNALING_PATHWAY & 79 & -2.304 & $<0.001$ \\
\hline
\end{tabular}

TCGA-CM cohort. Significantly, we found RNA polymerase and acid metabolism pathways to be activated in the highrisk group (Figure 5(a), Table 1), while in the low-risk group, which means high-expressed FCRLs, the following immuneassociated pathways were activated: natural killer cell- (NK-) mediated cytotoxicity, leukocyte transendothelial migration, T cell receptor signaling pathway, and B cell receptor signaling pathway (Figure 5(b), Table 1).

3.5. FCRLs Affect the Progress of CM through B Cells. Based on the results of GSEA, we evaluated the connection between FCRLs and immune cells with TIMER. As shown in Figure 6, we can see that all the FCRL1-6 were significantly positively linked with B cells, CD8+ T cells, CD4+ $\mathrm{T}$ cells, neutrophils, and dendritic cells (all, $P<0.05$ ), while not being significantly positively linked with macrophages. The association between FCRL1-6 and B cells is the most significant, and all the $R$-values are almost higher than 0.3 , so we also analyzed the association between FCRLs and two marker genes of B cell, CD19 and CD79A. As shown in Figure 7, all the FCRL1-6 are both highly correlated with CD19 and CD79A among all the CM patients (all, $P<0.05, R>0.6$ ).

\section{Discussion}

$\mathrm{CM}$ is a common immune-related malignancy in the West, causing the majority (75\%) of deaths related to skin cancer $[8,28]$. Many kinds of research have revealed that an intact immune system can prevent/control and shape/promote cancer $[29,30]$. The effects of FCRLs on the immune system have been discovered in some autoimmune disease, such as Graves' disease [21], systemic lupus erythematosus [23], and rheumatoid arthritis [31]. Rostamzadeh et al. [32] also demonstrated that FCRL overexpression in B cell malignancies introduce them as logical candidates for the development of antibody- and cell-based immunotherapy approaches in B cell malignancies and immune-mediated and infectious diseases. The relationship between immune system response and CM may be achieved through FCRL2 and FCRLA [24, 33]; however, the overall prognostic value of FCRLs on the OS of CM has not been clearly demonstrated yet.

Our study hypothesized that the expression of FCRLs affects the outcome of CM. And the results associated with Breslow's depth revealed the expression of FCRL1-6 was lower in patients with a Breslow's depth of $3 \mathrm{~cm}$ or greater. Further results showed that the lower expression of FCRL1- 


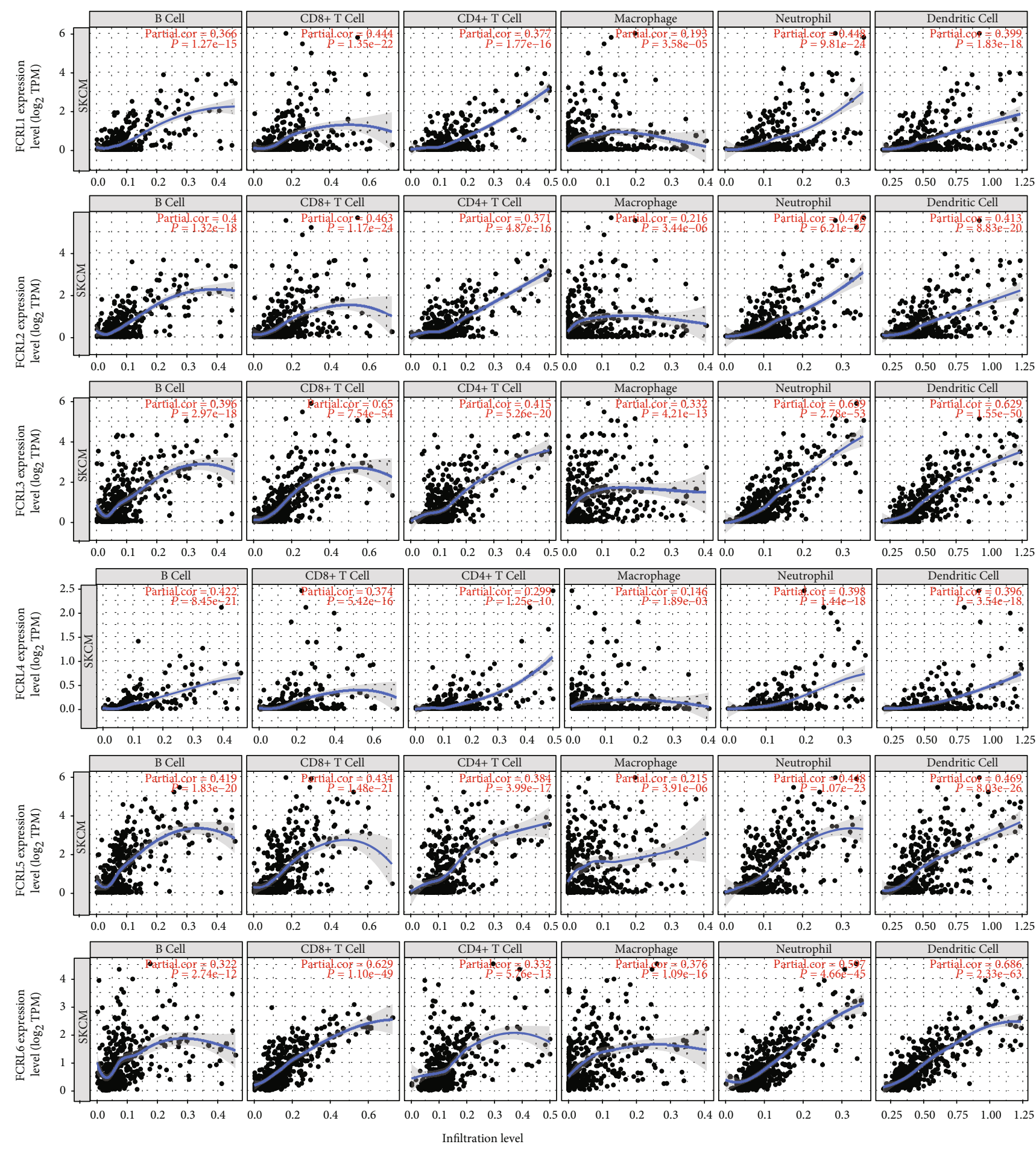

FIGURE 6: The association between FCRL1-6 and infiltration of tumor purity, B cells, CD8+ cells, CD4+ cells, macrophage, neutrophils, and dendritic cells. The FCRL1-6 expression level was displayed by the $\log _{2}$ RSEM value; Pearson's correlation analysis was used to assess the correlations. $P<0.05$ was considered significant difference.

6 all depicted the poor OS. Furthermore, as we evaluated the prognostic value of pathological and clinical features, we found that OS was worse in patients older than 60-year-old and pathological Stages III and IV. The overall prognostic value of FCRLs was confirmed in the TCGA-CM cohort and GSE65904 cohort. Based on our findings, we can con- clude that the expression of FCRL1-6 is beneficial to predict the prognosis of CM patients.

To study the potential mechanism of how FCRLs affect the OS of CM patients, we conducted the GSEA analysis among the FCRL-determined high- and low-risk groups. We found that in the low-risk group, several immune- 

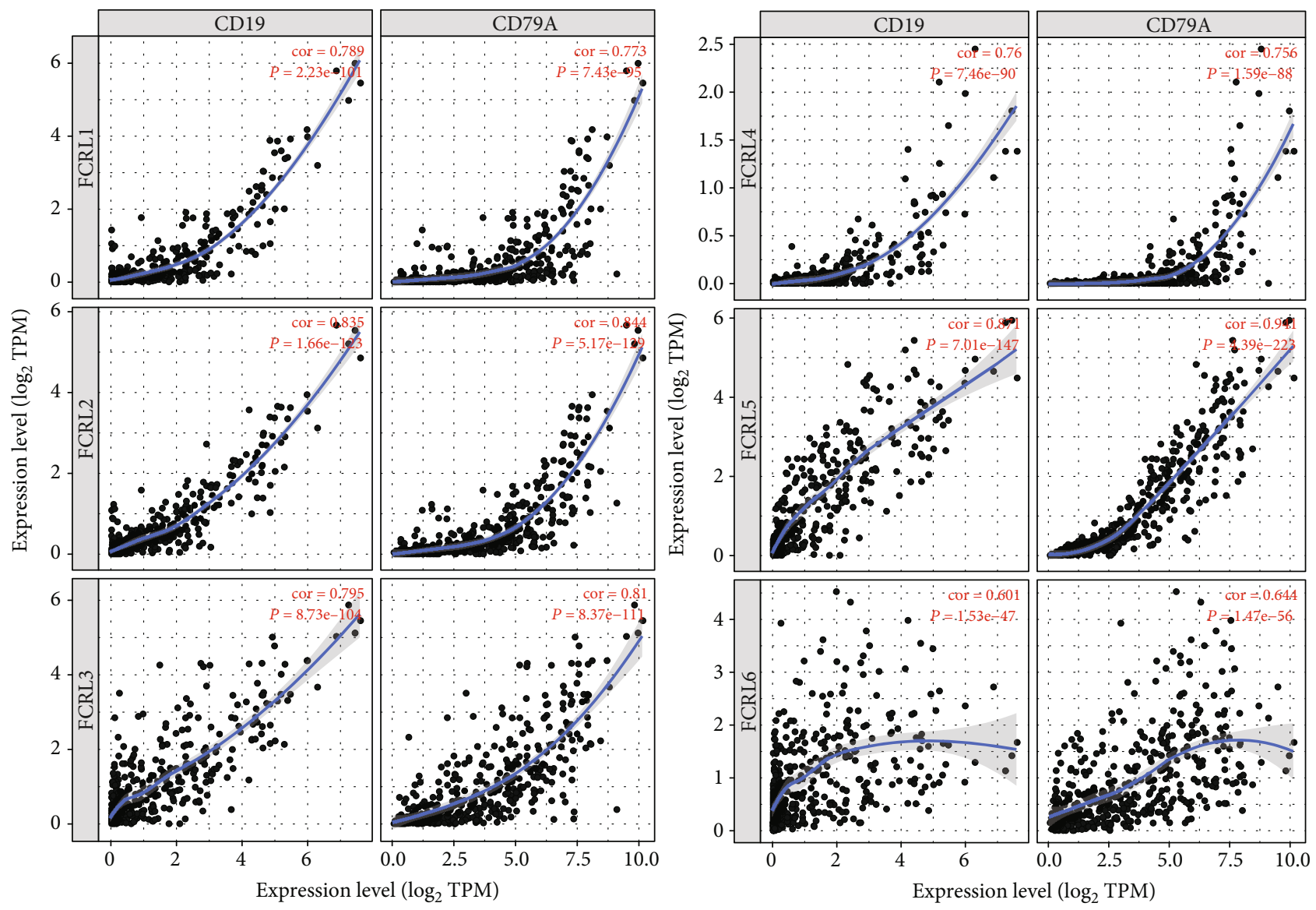

FIgure 7: The association between FCRL1-6 and B cell markers, CD19 and CD79A. The FCRL1-6 expression level was displayed by the log 2 RSEM value; Pearson's correlation analysis was used to assess the correlations. $P<0.05$ was considered significant difference.

related pathways were activated, including NK-mediated cytotoxicity, leukocyte transendothelial migration, $\mathrm{T}$ cell receptor signaling pathway, and $\mathrm{B}$ cell receptor signaling pathway. These activated pathways might act as the protect factors and associated with the favourable outcome of CM. We found some studies to prove the new findings. Baginska et al. [34] revealed that Beclin 1 could suppress the progression of tumors through facilitating NK-mediated tumor cell killing in breast and melanoma tumor in mice model. Therefore, we analyzed the association between FCRLs and immune cell infiltration in CM and found that all the FCRL1-6 are significantly associated with the infiltration rate of $\mathrm{B}$ cells, and the further steps confirmed these findings; the expression of FCRL1-6 is correlated with the expression of B cell markers, CD19 and CD79A. The result is consistent with the results of the study by Li et al. They demonstrate that FCRL3 has a potent costimulatory effect on Toll-Like Receptor 9- (TLR9-) mediated B cell activation but inhibits plasma cell differentiation as well [35]. Meanwhile, the association between B cells and CM was also be widely reported. Saul et al. [36] demonstrated that, in patients with metastatic CM, tumorinfiltrating B cells had increased B cell receptor class switching and affinity maturation, which means the presence of an active antigen-driven B cell response. Gilbert et al. reported that patient-derived $\mathrm{B}$ cells can kill melanoma cells in vitro by antibody-dependent cytotoxicity (ADCC) [37].
Based on our findings, some of the advantages of our research are worth highlighting. First and foremost, this is the first study to comprehensively describe the relationship between FCRLs and CM and analyze the prognostic value of several pathological and clinical features, such as ages, Breslow's depth, and tumor stages. Secondly, we used the TCGA-CM data to establish the predicted signature of these FCRL1-6 and validate the predict value of it in the GSE65904 cohort. Thirdly, the potential signaling pathways impacted by FCRLs were also exposed, and the NK-mediated cytotoxicity, leukocyte transendothelial migration, $\mathrm{T}$ cell receptor, and $\mathrm{B}$ cell receptor pathways might be the key FCRLaffected pathways which might benefit the outcome of CM patients, as well as the infiltration of B cells. All in all, based on the information presented in the current study, FCRLs can be used as biomarkers to predict the risk of disease progression and the OS for CM patients. Our results could help the clinical prognosis evaluation and help to choose the most adaptive treatment according to the expression level of FCRLs. On the other hand, we should also clearly recognize that our research has some limitations. Firstly, the effects of FCRLA and FCRLB on CM need to be further studied to obtain more comprehensive and accurate conclusions. Secondly, we could determine only how the FCRLs affected CM based on limited clinical data, such as tumor stage and OS, but we lack in-depth research on the impact of the 
environment, lifestyle, and other risk factors, so future research should consider other genetic-behaviors, genegene interactions to ensure a better and more comprehensive understanding of the relationship between the FCRLs and the risk of CM.

\section{Conclusion}

In conclusion, our study revealed that highly expressed FCRLs were associated with favourable OS in CM, with the exception of FCRLA and FCRLB. FCRL1-6-based prediction signature was established in TCGA-CM database and validated in GSE65904 and could act as a biomarker to predict the prognosis of patients with CM.

\section{Data Availability}

The data used to support the findings of this study are included within the article. The gene expression data can be accessed on The Cancer Genome Atlas (TCGA) and Gene Expression Omnibus (GEO).

\section{Conflicts of Interest}

The authors certify that they have no affiliations with or involvement in any organisation or entity with any financial interest or nonfinancial interest in the subject matter or materials discussed in this manuscript.

\section{Authors' Contributions}

Yu Liu and Yiding Chen contributed equally to this work.

\section{References}

[1] J. W. Little, "Melanoma: etiology, treatment, and dental implications," General dentistry, vol. 54, no. 1, pp. 61-66, 2006.

[2] M. Bigby, "Clinical dermatology: a color guide to diagnosis and therapy," Archives of Dermatology, vol. 132, no. 9, pp. 1136-1136, 1996.

[3] F. Bray, J. Ferlay, I. Soerjomataram, R. L. Siegel, L. A. Torre, and A. Jemal, "Global cancer statistics 2018: GLOBOCAN estimates of incidence and mortality worldwide for 36 cancers in 185 countries," CA: a Cancer Journal for Clinicians, vol. 68, no. 6, pp. 394-424, 2018.

[4] D. D. Yang, J. D. Salciccioli, D. C. Marshall, A. Sheri, and J. Shalhoub, "Trends in malignant melanoma mortality in 31 countries from 1985 to 2015," The British Journal of Dermatology, 2020.

[5] R. L. Siegel, K. D. Miller, and A. Jemal, "Cancer statistics, 2019,” CA: a Cancer Journal for Clinicians, vol. 69, no. 1, pp. 7-34, 2019.

[6] M. Situm, M. Buljan, S. O. Bulic, and D. Simic, "The mechanisms of UV radiation in the development of malignant melanoma," Collegium antropologicum, vol. 31, Supplement 1, pp. 13-16, 2007.

[7] K. Volkovova, D. Bilanicova, A. Bartonova, S. Letasiova, and M. Dusinska, "Associations between environmental factors and incidence of cutaneous melanoma. Review," Environmental Health, vol. 11, Supplement 1, 2012.
[8] G. Palmieri, M. Ombra, M. Colombino et al., "Multiple molecular pathways in melanomagenesis: characterization of therapeutic targets," Frontiers in Oncology, vol. 5, 2015.

[9] S. C. S. Simon, X. Hu, J. Panten et al., "Eosinophil accumulation predicts response to melanoma treatment with immune checkpoint inhibitors," Oncoimmunology, vol. 9, no. 1, article 1727116, 2020.

[10] M. Gil and K. E. Kim, "Interleukin-18 is a prognostic biomarker correlated with CD8(+) T cell and natural killer cell infiltration in skin cutaneous melanoma," Journal of clinical medicine, vol. 8, no. 11, 2019.

[11] S. L. Park, A. Buzzai, J. Rautela et al., "Tissue-resident memory CD8(+) $\mathrm{T}$ cells promote melanoma-immune equilibrium in skin," Nature, vol. 565, no. 7739, pp. 366-371, 2019.

[12] A. M. Eggermont, V. Chiarion-Sileni, J. J. Grob et al., "Prolonged survival in stage III melanoma with ipilimumab adjuvant therapy," The New England Journal of Medicine, vol. 375, no. 19, pp. 1845-1855, 2016.

[13] R. S. Davis, "Fc receptor-like molecules," Annual Review of Immunology, vol. 25, no. 1, pp. 525-560, 2007.

[14] G. R. Ehrhardt, C. M. Leu, S. Zhang et al., "Fc receptor-like proteins (FCRL): immunomodulators of B cell function," Advances in Experimental Medicine and Biology, vol. 596, pp. 155-162, 2007.

[15] G. Daniele, A. L'Abbate, A. Turchiano et al., "1q23.1 homozygous deletion and downregulation of $\mathrm{Fc}$ receptor-like family genes confer poor prognosis in chronic lymphocytic leukemia," Clinical and Experimental Medicine, vol. 19, no. 2, pp. 261-267, 2019.

[16] T. Kazemi, H. Asgarian-Omran, M. Hojjat-Farsangi et al., "Fc receptor-like 1-5 molecules are similarly expressed in progressive and indolent clinical subtypes of B-cell chronic lymphocytic leukemia," International Journal of Cancer, vol. 123, no. 9, pp. 2113-2119, 2008.

[17] T. Kazemi, H. Asgarian-Omran, A. Memarian et al., "Low representation of Fc receptor-like 1-5 molecules in leukemic cells from Iranian patients with acute lymphoblastic leukemia," Cancer Immunology, Immunotherapy, vol. 58, no. 6, pp. 989996, 2009.

[18] K. Wang, H. Pei, B. Huang et al., "Overexpression of fc receptor-like 1 associated with B-cell activation during hepatitis B virus infection," Brazilian Journal of Medical and Biological Research, vol. 45, no. 12, pp. 1112-1118, 2012.

[19] S. Portugal, C. M. Tipton, H. Sohn et al., "Malaria-associated atypical memory B cells exhibit markedly reduced B cell receptor signaling and effector function," Elife, vol. 4, article e07218, 2015.

[20] M. F. Muellenbeck, B. Ueberheide, B. Amulic et al., "Atypical and classical memory B cells produce plasmodium falciparum neutralizing antibodies," The Journal of Experimental Medicine, vol. 210, no. 2, pp. 389-399, 2013.

[21] D. Rostamzadeh, M. H. Dabbaghmanesh, M. Shabani, A. Hosseini, and Z. Amirghofran, "Expression profile of human fc receptor-like 1,2 , and 4 molecules in peripheral blood mononuclear cells of patients with Hashimoto's thyroiditis and Graves' disease," Hormone and Metabolic Research, vol. 47, no. 9, pp. 693-698, 2015.

[22] L. Yeo, H. Lom, M. Juarez et al., "Expression of FcRL4 defines a pro-inflammatory, RANKL-producing B cell subset in rheumatoid arthritis," Annals of the Rheumatic Diseases, vol. 74, no. 5, pp. 928-935, 2015. 
[23] K. O. Baranov, O. Volkova, L. V. Mechetina et al., "Expression of human B-cell specific receptor FCRL1 in normal individuals and in patients with autoimmune diseases," Molekuliarnaia biologiia, vol. 46, no. 3, pp. 500-507, 2012.

[24] N. A. Chikaev, E. A. Bykova, A. M. Najakshin et al., "Cloning and characterization of the human FCRL2 gene," Genomics, vol. 85, no. 2, pp. 264-272, 2005.

[25] A. Subramanian, P. Tamayo, V. K. Mootha et al., "Gene set enrichment analysis: a knowledge-based approach for interpreting genome-wide expression profiles," Proceedings of the National Academy of Sciences of the United States of America, vol. 102, no. 43, pp. 15545-15550, 2005.

[26] R. C. Gentleman, V. J. Carey, D. M. Bates et al., "Bioconductor: open software development for computational biology and bioinformatics," Genome biology, vol. 5, no. 10, 2004.

[27] T. Li, J. Fan, B. Wang et al., "TIMER: a web server for comprehensive analysis of tumor-infiltrating immune cells," Cancer Research, vol. 77, no. 21, pp. e108-e110, 2017.

[28] D. Schadendorf and A. Hauschild, "Melanoma in 2013: melanoma-the run of success continues," Nature Reviews. Clinical Oncology, vol. 11, no. 2, pp. 75-76, 2014.

[29] V. Shankaran, H. Ikeda, A. T. Bruce et al., "IFNgamma and lymphocytes prevent primary tumour development and shape tumour immunogenicity," Nature, vol. 410, no. 6832, pp. 1107-1111, 2001.

[30] G. P. Dunn, C. M. Koebel, and R. D. Schreiber, "Interferons, immunity and cancer immunoediting," Nature Reviews. Immunology, vol. 6, no. 11, pp. 836-848, 2006.

[31] A. Khanzadeh, Z. Habibagahi, A. Hosseini, and Z. Amirghofran, "Investigation of the human FCRL1, 2, and 4 gene expressions in patients with rheumatoid arthritis," Rheumatology International, vol. 36, no. 8, pp. 1149-1156, 2016.

[32] D. Rostamzadeh, T. Kazemi, Z. Amirghofran, and M. Shabani, "Update on Fc receptor-like (FCRL) family: new immunoregulatory players in health and diseases," Expert Opinion on Therapeutic Targets, vol. 22, no. 6, pp. 487-502, 2018.

[33] T. Inozume, Y. Matsuzaki, S. Kurihara et al., "Novel melanoma antigen, FCRL/FREB, identified by cDNA profile comparison using DNA chip are immunogenic in multiple melanoma patients," International Journal of Cancer, vol. 114, no. 2, pp. 283-290, 2005.

[34] J. Baginska, E. Viry, G. Berchem et al., "Granzyme B degradation by autophagy decreases tumor cell susceptibility to natural killer-mediated lysis under hypoxia," Proceedings of the National Academy of Sciences of the United States of America, vol. 110, no. 43, pp. 17450-17455, 2013.

[35] F. J. Li, D. M. Schreeder, R. Li, J. Wu, and R. S. Davis, "FCRL3 promotes TLR9-induced B-cell activation and suppresses plasma cell differentiation," European Journal of Immunology, vol. 43, no. 11, pp. 2980-2992, 2013.

[36] L. Saul, K. M. Ilieva, H. J. Bax et al., "IgG subclass switching and clonal expansion in cutaneous melanoma and normal skin," Scientific Reports, vol. 6, no. 1, article 29736, 2016.

[37] A. E. Gilbert, P. Karagiannis, T. Dodev et al., "Monitoring the systemic human memory B cell compartment of melanoma patients for anti-tumor IgG antibodies," PLoS One, vol. 6, no. 4 , article e19330, 2011. 